\title{
Review of: "A nicotinic hypothesis for Covid-19 with preventive and therapeutic implications"
}

\section{Steven Ubaldo Alvarez}

Potential competing interests: The author(s) declared that no potential competing interests exist.

Well written paper on the regulation of ace2. Let's get those synthetic nicotine patches made without the addictive effects manufactured and sell them as anti-Sar2-Covid-19 patches for the masses. We can do this Earth . 